Poincare Journal of Analysis E Applications

Vol. 2015 (2), Special Issue (IWWFA-II, Delhi), 37-52

CPoincare Publishers

\title{
MULTIRESOLUTION ANALYSES ON QUASILATTICES
}

\author{
Wayne M. Lawton
}

\author{
Date of Receiving : $\quad$ 01.04.2015 \\ Date of Revision : 05. 05. 2015 \\ Date of Acceptance : 06.05 .2015
}

\begin{abstract}
We derive relations between geometric means of the Fourier moduli of a refinable distribution and of a related polynomial. We use Pisot-Vijayaraghavan numbers to construct families of one dimension quasilattices and multiresolution analyses spanned by distributions that are refinable with respect to dilation by the PV numbers and translation by quasilattice points. We conjecture that scalar valued refinable distributions are never integrable, construct piecewise constant vector valued refinable functions, and discuss multidimensional extensions.
\end{abstract}

\section{Refinable Distributions}

In this paper $\mathbb{Z}, \mathbb{N}=\{1,2,3, \ldots\}, \mathbb{Q}, \mathbb{R}, \mathbb{C}$ denote the integer, natural, rational, real, and complex numbers and $\mathbb{T}=\mathbb{R} / \mathbb{Z}$ denotes the circle group. Let $\alpha_{1}, \alpha_{2}, \ldots$ be a sequence of positive real numbers, let $\mu_{1}, \mu_{2}, \ldots$ be a sequence of complex probability measures whose total variations are uniformly bounded and whose supports satisfy $\operatorname{supp}\left(\mu_{n}\right) \subset$ $\left[-\alpha_{n}, \alpha_{n}\right]$, and define the sequence of complex probability measures $f_{n}=\mu_{1} * \cdots * \mu_{n}$ where * denotes convolution. Our result ([28], Theorem 2.1) implies that if $\alpha_{j} \rightarrow 0$ exponentially fast then $f_{n}$ converges to a compactly supported distribution. Our result addresses general Lie groups and our proof avoids Fourier transforms. In [57] Uchida proved that $f_{n}$ converges to a hyperfunction under the much weaker assumption that $\sum_{n} \alpha_{n}<\infty$. His result addresses commutative Lie groups and his proof uses the Fourier transform.

Remark 1.1. The complex Fourier transforms $\widehat{f}_{n}(z)=\int_{-\infty}^{\infty} f_{n}(x) e^{2 \pi i x z} d x=\prod_{j=1}^{n} \widehat{\mu}_{j}(z)$ and if $\alpha_{n} \rightarrow 0$ exponentially fast then the argument that Daubechies credits to Deslauriers and Dubuc [13] and uses to prove ([10], Lemma 6.2.2) shows that $\widehat{f_{n}}(z)$ converges to an entire function of exponential type which the Paley-Wiener Theorem ([15], Section 3.3), [39] shows is equal to the Fourier transform $\widehat{f}$ of a compactly supported distribution $f$.

Let $\lambda \in \mathbb{R} \backslash[-1,1], a_{1}, \ldots . a_{m} \in \mathbb{C} \backslash\{0\}, a_{1}+\cdots+a_{m}=|\lambda|, \tau_{1}<\cdots<\tau_{m} \in \mathbb{R}$, and define the sequence of complex probability measures by $\mu_{n}=|\lambda|^{-1} \sum_{j=1}^{m} a_{j} \delta_{\tau_{j} / \lambda^{n}}$ where for $r \in \mathbb{R}, \delta_{r}$ denotes the Dirac point measure located at $r$. Since $\operatorname{supp}\left(\mu_{n}\right) \subset\left[\tau_{1} / \lambda^{n}, \tau_{m} / \lambda^{n}\right]$

2010 Mathematics Subject Classification. 11R06, 42C15, 47A68.

Communicated by. Richard A. Zalik 